\title{
Hallazgos radiográficos de rutina en odontología, y su importancia en el diagnóstico precoz de patologías cervicofaciales. Reporte de caso
}

\section{Routine radiographic findings in dentistry, and their importance in the early diagnosis of cervicofacial pathologies. Case report.}

\author{
Aguilar-Maldonado, José David ${ }^{1 *}$, Duchi-Valdez, Jessica Paola ${ }^{1}$, Vivar-Inga, Vanessa Estefania ${ }^{1}$ y Terreros, \\ Andrea \\ ${ }^{1}$ Universidad Católica de Cuenca \\ *jdaguilarm@ucacue.edu.ec
}

DOI: https://doi.org/10.26871/killcana_salud.v2i1.174

\begin{abstract}
Resumen
Contexto: La radiografía panorámica, ortopantomografía o tomografía panorámica dental es una técnica radiográfica muy utilizada en odontología. Y dentro de las patologías cervicofaciales encontradas en estas radiografías de rutina están las placas ateromatosas calcificadas, originadas por el depósito excesivo de lípidos y células inflamatorias, ocasionando el posible cierre de una arteria en su pared íntima. Objetivo: El objetivo de este artículo es enfatizar la importancia del diagnóstico a nivel cervicofacial en ortopantomografías de rutina en la práctica odontológica. Materiales y Métodos: Este artículo presenta el caso de una paciente femenina de 42 años, quien acude al centro de especialidades odontológicas de la Universidad Católica De Cuenca sede Azogues, para un control de su salud bucal, se le realizó una ortopantomografía diagnostica de rutina, en la cual presento radiopacidades a nivel cervical, los cuales fueron diagnosticados como placas ateromatosas en carótida. Resultados:Esta patología se cronifica por la presencia de factores de riesgo que ocasionan un daño endotelial, tales como: hipertensión arterial, diabetes mellitus, obesidad entre otros. El diagnóstico de esta patología es de relevancia ya que ayudará en la prevención de enfermedades como el accidente cerebro vascular e infarto cerebral. Conclusiones: Durante la atención odontológica rutinaria es indispensable realizar una buena lectura radiográfica y una adecuada anamnesis ya que podemos encontrar datos muy importantes sobre la salud en general del paciente llevándonos a diagnosticar precozmente posibles patologías.
\end{abstract}

Palabras clave: Ortopantomografia, Ateroma, Carótida.

\begin{abstract}
Context: Panoramic radiography, orthopantomography, or dental panoramic tomography, is a popular radiographic technique in dentistry. Among the cervicofacial pathologies found in these routine $x$-rays are the calcified atheromatous plaques which are originated by the excessive deposit of lipids and inflammatory cells which are the possible causes of closure at the inner layer of the arterial wall. Objective: The objective of this article is to emphasize the importance of cervical-facial diagnosis, in routine orthopantomographies, in dental practice. Materials and Methods: This article presents the case of a 42 year old female patient, who goes to the Dental Specialty Center of the Catholic University of Cuenca in Azogues for her oral health examination. She underwent a routine diagnostic orthopantomography which revealed that she presents radiopacities at the cervical level, these were diagnosed as carotid atheromatous plaques. Results: This pathology becomes chronic with the presence of risk factors that cause endothelial damage, such as: high blood pressure, diabetes mellitus and obesity, among others. The diagnosis of this pathology is relevant as it will help in the prevention of diseases such as the cerebrovascular accident and ischemic stroke. Conclusions: During routine dental care, it is essential to carry out a good radiographic reading and a proper anamnesis since we can find very important data about the patient's general health, leading us to diagnose possible pathologies at an early stage.
\end{abstract}

Key words: Orthopantomography, Atheroma, Carotid.

\section{Introducción}

La radiografía panorámica, ortopantomografía o tomografía panorámica dental es una técnica radiográfica muy utilizada en odontología. Esta técnica permite al profesional observar áreas del maxilar y la mandíbula, espacios de aire, y tejidos blandos en una sola proyección. Sin embargo, 
es necesario que el profesional odontólogo tenga conocimiento básico de las señales anatómicas normales para interpretar las imágenes panorámicas y posibles patologías cervicofaciales que puedan ser diagnosticadas tempranamente. $^{1,2}$

Una de estas patologías es la arterioesclerosis, una enfermedad crónica producida por el depósito de células inflamatorias y lípidos que se calcifica en la pared íntima arterial seguido de una apoptosis por parte de las células musculares lisas y por una liberación de vesículas de la matriz. El resultado de la confluencia y fragmentación originarán placas calcificadas, llamadas placas de ateroma, las cuales se propagan a través de una matriz de tejido colágeno en la cual formarán placas fibrocalcificadas que finalmente podría cerrar la luz de las arterias. ${ }^{3-5}$

Clínicamente se presenta en forma de cardiopatía isquémica, enfermedad arterial coronaria, enfermedad cerebrovascular (ACV) e infarto cerebral., 6

Esta patología está relacionada con individuos de edad avanzada, el incremento de la presión arterial, los altos niveles séricos de carbohidratos o colesterol así como otros factores como el tabaquismo, diabetes mellitus y la menopausia que pueden conducir al desarrollo de daño endotelial y aterogénesis. ${ }^{3,4}$

Existen diferentes métodos de diagnóstico para la ateroesclerosis como: el ultrasonido, Eco Color Doppler, exámenes imagenológicos (Rx panorámica), la angioresonancia magnética, la tomografía computarizada (TC) y la angiografía invasiva por sustracción digital, considerada como el gold standard y es el método utilizado en la mayoría de los ensayos clínicos. ${ }^{3,6,7}$

El tratamiento para la ateroesclerosis se basa en terapias médicas con fármacos y tratamientos quirúrgicos como, la endarterectomía carotidea y la angioplastia carotidea. ${ }^{6,7}$

\section{Etiología}

Las placas de ateroma se originan a partir de una disfunción endotelial (DE). La DE es un desequilibrio de las sustancias producidas por el endotelio que induce al incremento de la permeabilidad vascular, la inflamación y la vasoconstricción, y que puede favorecer el desarrollo de arteriosclerosis, agregación plaquetaria y trombosis. ${ }^{8}$

Además, existen factores de riesgo que pueden producir DE tales como: el colesterol unido a lipoproteínas de baja densidad (cLDL), el tabaquismo, diabetes, hipertensión, obesidad, menopausia, enfermedad renal crónica, etc. Y otros factores emergentes (radicales libres de oxígeno, homocisteína, infecciones, déficit estrogénico, etc. $)^{8-10}$

Si la DE evoluciona, puede producirse la rotura de la barrera endotelial favoreciendo el depósito de partículas lipídicas en la pared arterial, seguido de apoptosis de las células musculares lisas y de la liberación de las vesículas de la matriz. Dicho proceso, junto con la infiltración de macrófagos, inicia con la deposición de sales de calcio que producen la calcificación de la íntima de la pared arterial. La desintegración y afluencia de las placas calcificadas se propaga a través de una matriz de tejido colágeno y forma las placas fibrocalcificadas., ${ }^{4,9}$

Las arterias afectadas son la aorta, coronarias y cerebrales, incluyendo la arteria carótida. ${ }^{9}$

\section{Localización}

Las placas de ateroma pueden estar ubicadas en las superficies adyacentes a los tejidos del cuello (principalmente donde se inicia la bifurcación de la arteria carótida externa e interna, inferior y lateralmente al hueso hioides) $)^{11}$

Surur et al. 2014, refiere que la región más afectada es el sifón carotideo y el segmento vertebral $\mathrm{C} 4$, mientras que las arterias cerebrales mostraron un compromiso escaso. Además, se observó una alta incidencia en pacientes mayores a 55 años, sin predominio en algún sexo; aunque otros estudios mencionan que el sexo masculino es el más afectado. También prevaleció en pacientes de raza blanca, por lo que no se pudo determinar la incidencia en la raza negra. $^{12}$

\section{Características histológicas}

Es un tipo de lesión conocida como lesión avanzada debido a la desorganización de la íntima. Estas placas ateromatosas están constituidas principalmente de macrófagos, lípidos extracelulares, calcio, tejido conectivo fibroso, etc., 11,13

\section{Características radiográficas}

Radiográficamente las placas de ateroma se detectan como masas nodulares heterogéneas o como líneas verticales radiopacas unilaterales o bilaterales, por la calcificación de las mismas, siguiendo el trayecto del vaso. La localización generalmente suele ser por debajo del ángulo de la mandíbula, a nivel del espacio de la tercera vértebra cervical (entre C3 y C4) a nivel o por debajo del hueso hioides., ${ }^{3,9}$

\section{Diagnóstico diferencial}

Al interpretar una radiografía panorámica, se debe tener en cuenta las formas anatómicas y patológicas como diagnóstico diferencial, ya que también reproducen radiopacidades y pueden producir confusiones diagnósticas. Dentro de las anatómicas están el cartílago tritíceo, hueso hioides, calcificación de los ligamentos estilo hioideo y estilo mandibular, cartílago tiroides calcificado, la apófisis estiloides, el lóbulo auricular, las vértebras, el tubérculo anterior del atlas, la lengua, la epiglotis y el paladar blando. Entre las patológicas están los nódulos linfáticos calcificados, flebolitos, sialolitos, cuerpos libres, tonsolitos y acné calcificado. . $^{3,9}$ 


\section{Tratamiento médico}

En un inicio es relevante controlar los factores de riesgo, debido a que la presencia de tabaquismo, consumo de alcohol, obesidad, hipertensión arterial (HTA), y diabetes mellitus que podrían influir en la obliteración arterial. Además, es recomendable valorar la actividad física y la dieta. Si la modificación del estilo de vida falla, se emplea un tratamiento farmacológico como segunda elección, sobre todo en los enfermos con hipertensión, hiperglucemias e hiperlipidemia. ${ }^{14}$

Los fármacos indicados para el tratamiento son los inhibidores de la enzima de conversión de la angiotensina (IECA) para el control de la HTA, antidiabéticos orales o insulina para la diabetes, estatinas para el manejo de la dislipemia y antiagregación mediante la administración de aspirina en dosis bajas, triflusal o clopidogrel. ${ }^{7}$

\section{Tratamiento quirúrgico}

La endarterectomía es un procedimiento quirúrgico que consiste en seccionar una arteria (arterioctomía), inicialmente se realiza la remoción de la placa ateromatosa. Se debe realizar endarterectomía en pacientes sintomáticos con estenosis superiores al $70 \%$ y con estenosis entre el $50-69 \%$, con menor beneficio y solo en pacientes seleccionados siempre que la tasa de morbimortalidad quirúrgica sea inferior a $5 \% .^{6,14}$

Otro procedimiento quirúrgico terapéutico es la angioplastia arterial, el cual es un procedimiento endovascular, el mismo que a menudo suele ir acompañado de implantación de stents. Por lo tanto, es una técnica alternativa a la endarterectomía. ${ }^{14}$

El objetivo de la presentación de este caso clínico es enfatizar en la importancia del diagnóstico a nivel cervicofacial en ortopantomografías de rutina en la práctica odontológica.

\section{Caso clínico}

Paciente de sexo femenino de 42 años de edad, acudió al centro de Especialidades Odontológicas de la Clínica I, de la Universidad Católica de Cuenca sede Azogues, Ecuador, para una revisión de su estado bucodental de rutina, donde además refirió dolor constante y pulsátil en la pieza dental 2.5 .

En antecedentes personales la paciente refirió presentar asma, hipertensión y diabetes controladas; tres embarazos de los cuales 2 llegaron a término, refiere ocupación laboral que la expone constantemente a la inhalación de humo. Como antecedentes médicos familiares manifestó madre diabética y con episodio de trombosis reciente.

Dentro del examen físico general se presenta con signos de obesidad, facies de fatiga, y se moviliza por sus propios medios, los signos vitales se encontraron dentro de los rangos de normalidad, sin evidencia de ganglios palpables en región cervicomandibular, al examen clínico intraoral se observó edentulismo parcial bimaxilar, caries dental, enfermedad periodontal moderada (fig. 1) restauraciones dentales en mal estado, acumulación de detritus y placa bacteriana, además de presencia de cálculos dentales. (fig. 2)

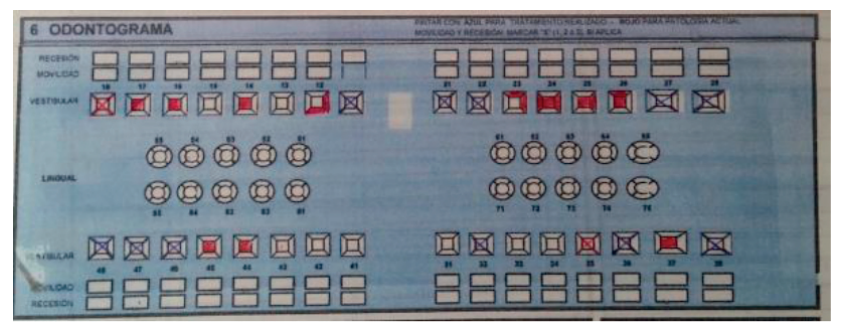

Fig. 1. Odontograma.

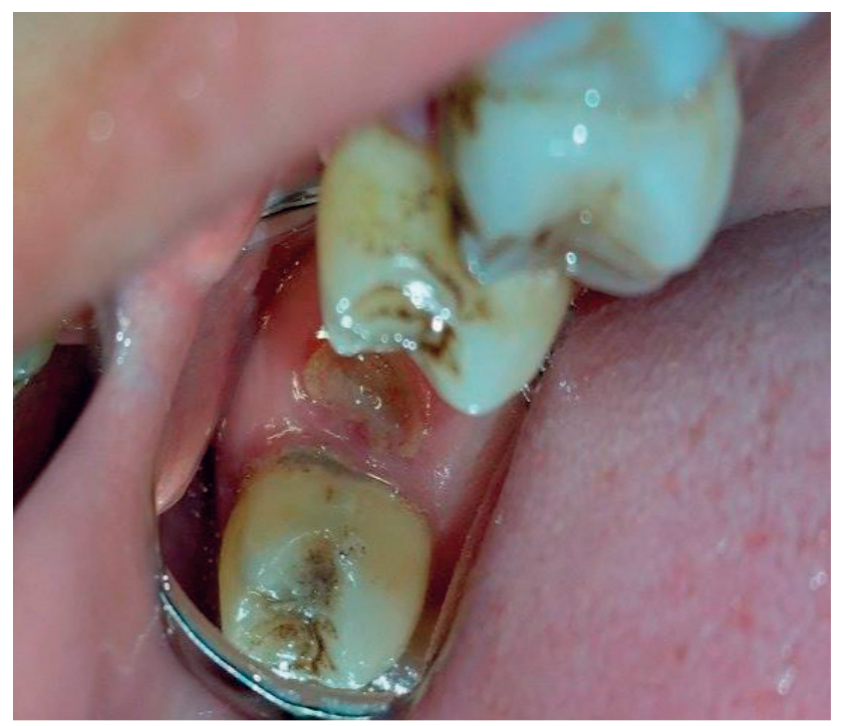

Fig. 2. Se observa resto radicular, placa bacteriana, cálculos dentales, pigmentaciones y mal estado de periodonto.

Al realizar el examen radiográfico diagnóstico, mediante una ortopantomografía se observaron varias lesiones cariosas, restos radiculares, pérdida de soporte óseo, ligera expansión del espacio sinusal izquierdo, asimetría condilar (fig. 3).

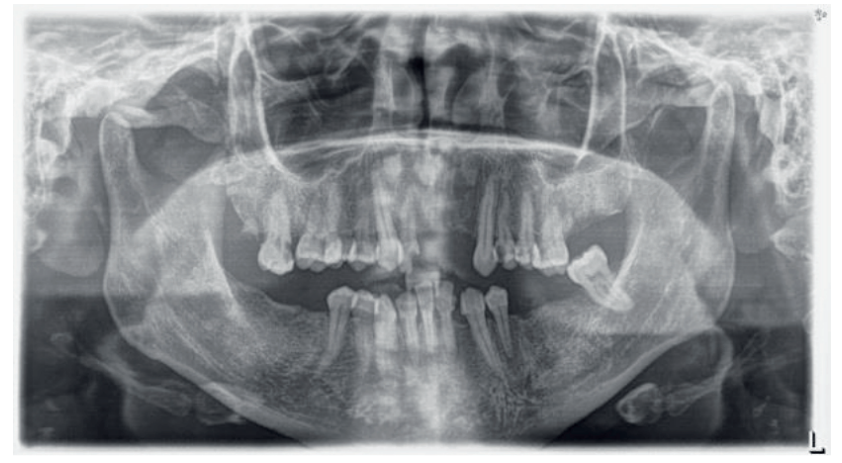

Fig. 3. Ortopantomografía que muestra estado deficiente del sistema estomatognático. 
Como hallazgo inusual se detectó la presencia de 3 radiopacidades a nivel de la cuarta vértebra cervical $(\mathrm{C} 4)$ con morfología irregular, bilaterales de densidad similar al hueso compacto, que midieron: $2.2 \mathrm{~mm} \mathrm{x} 1 \mathrm{~mm} ; 2 \mathrm{~mm} x$ $0.8 \mathrm{~mm} ; 0.6 \mathrm{~mm} \times 0.4 \mathrm{~mm}$ respectivamente. (fig. 4) Las cuales guían a un diagnóstico presuntivo de placas de ateroma en la arteria carótida $v s$ sialolitos en glándula submaxilar $v s$ calcificación distrófica de ganglios cervicales.

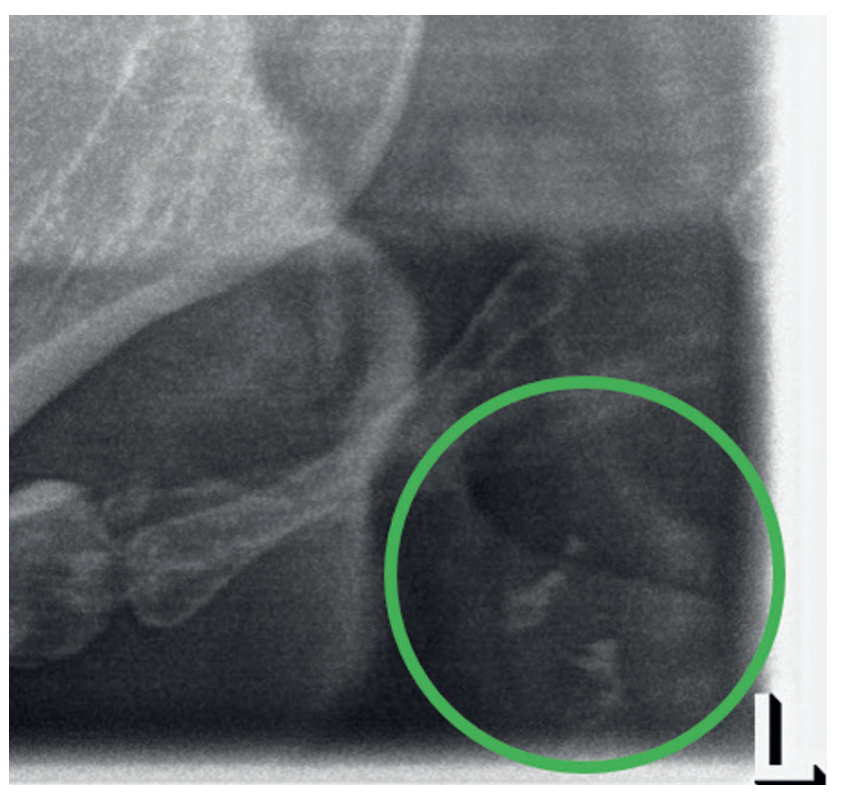

Fig. 4. Radiopacidades irregulares a nivel de C5

\section{Discusión}

Es de importancia que el profesional odontólogo sepa diferenciar imágenes radiopacas de estructuras anatómicas o patológicas en una ortopantomografía de rutina en la consulta, positivas ante un diagnóstico de placas de ateroma.

Salazar G y et al. $(2011)^{5}$ realizaron un estudio descriptivo transversal donde evaluaron 96 radiografías panorámicas de pacientes mayores a 43 años, relatando un $(3,07 \%)$ de calcificaciones, como masas nodulares radiopacas justo debajo del espacio intervertebral entre $\mathrm{C} 3$ y $\mathrm{C} 4$.

Santos JMO y cols. (2018) interpretaron 2500 radiografías panorámicas digitales en el estado de Ceará, Brasil, mencionando que el $4 \%(n=96)$ de pacientes presentaron posibles calcificaciones de la carótida, de los cuales el $40.4 \%$ fueron biliaterales, con predominio al sexo femenino $^{15}$

Ramos, A. y cols (2018) también indica que un 7,92\% fueron posibles calcificaciones, el $8.8 \%$ fue para el sexo femenino del grupo estudiado y además se asociaron con la edad, la hipertensión y los hábitos de tabaquismo. ${ }^{16}$

Monteiro IA, y cols. (2018) encontraron calcificaciones en un $6.8 \%$, entre ellos el $8.18 \%$ pertenece a mujeres y el $4.4 \%$ fueron bilaterales. ${ }^{13}$ Mientras que Moshfeghi M y cols. (2014) revisaron 2014 ortopantomografías de pacientes y concluyeron que el 9,5\% tenían placas ateroscleróti- cas calcificadas, también menciona que las mujeres tienen un mayor riesgo de presentar aterosclerosis detectadas en la radiografía que los hombres, además se ve asociado con el aumento de la edad y condiciones como el accidente cerebrovascular y la hipertensión. ${ }^{11}$

Nasseh I y cols. (2018) determinaron que el $21.2 \%$ de los individuos con hipertensión presentaban imágenes de calcificación de la arteria carótida. ${ }^{17}$

Todos los estudios mencionados presentan gran significado estadístico y coincidencias semejantes en relación al sexo, edad y factores predisponentes que se asocian con la presencia de placas de ateromas.

En el presente caso la imagen radiolúcida bilateral resultó ser una placa de ateroma gracias al examen Doppler, tomando especial asociación los factores de riesgo de la paciente, tales como la hipertensión arterial, diabetes, obesidad así como su ocupación; y también la historia médica familiar, pues la madre presentó un episodio de trombosis posiblemente por una placa de ateroma calcificada no diagnosticada, lo que llevaría a sospechar de una asociación genética.

Además, es de gran relevancia que la anamnesis sea exhaustiva, debido a que puede revelar datos importantes sobre la salud sistémica del paciente, e incluso los antecedentes médicos familiares podrían llevar a realizar un diagnóstico presuntivo. Además, patologías bucodentales como la enfermedad periodontal se han relacionado con presencia de placas calcificadas a nivel de carótida.

\section{Conclusión}

Durante la atención odontológica rutinaria es indispensable realizar una buena lectura radiográfica y una adecuada anamnesis ya que se pueden encontrar datos muy importantes sobre la salud en general del paciente llevando a diagnosticar precozmente posibles patologías. En el presente caso, son los antecedentes personales y familiares lo que llevó a un diagnóstico presuntivo de placas de ateroma bilaterales, diagnóstico que se comprobó mediante el uso de Doppler. Por lo antes mencionado es necesario remitir al paciente a un médico para confirmar los resultados y determinar la extensión real de la enfermedad y con ello poder otorgar un adecuado tratamiento transdisciplinario.

\section{Abreviaturas y acrónimos \\ ACV Accidente cerebro vascular.}

cLDL Colesterol unido a lipoproteínas de baja densidad.

DE Daño endotelial.

HTA Hipertensión arterial.

IECA Inhibidores de la enzima de conversión de la angiotensina.

TC Tomografía Computarizada. 


\section{Referencias Bibliográficas}

1. Whaites E. Radiología odontológica: 2a ed. Buenos Aires: Médica Panamericana; 2010. OCLC: 804885718.

2. Iannucci JM, Howerton LJ. Radiografía dental: principios y técnicas. Caracas: Amolca; 2013. OCLC: 934392913.

3. Alven Arreaza, Marisabel Lara. Ateroma calcificado en carótida y radiografía panorámica: reporte de caso. Acta Odontológica Venezolana. 2011;49(3). Available from: https://www.actaodontologica.com/ediciones/2011/ 3/art-15/.

4. Rodríguez-Palomares JF, Evangelista Masip A. Cuantificación del calcio aórtico y arteriosclerosis vascular en individuos asintomáticos: más allá de las arterias coronarias. Revista Española de Cardiología. 2016;69(09):813-816. Available from: http://www.revespcardiol.org/es/cuantificacion-delcalcio-aortico-arteriosclerosis/articulo/90458136/ER.

5. Moranth GSAyFPEyRV. Detección de placas de ateroma calcificadas en la arteria carotida en la radiografía panorámica. Revista Colombiana de Investigación en Odontología. 2011;2(5):104-113. Available from: https: //www.rcio.org/index.php/rcio/article/view/56.

6. Sposato LA, Riccio PM, Klein FR. Diagnóstico y tratamiento de la enfermedad carotídea aterosclerótica extracraneal asintomática. Medicina (Buenos Aires). 2011 Dec;71(6):561-565. Available from: http://www.scielo.org.ar/scielo.php?script=sci_abstract\& pid=S0025-76802011000800014.

7. Consenso de Estenosis Carotídea. Sociedad Argentina de Cardiología; 2006. [Online; accessed 29. May 2018]. Available from: http://www.scielo. org.ar/scielo.php?script=sci_arttext \&pid=S185037482006000300014\&lng=en\&nrm=iso\&tlng=es.

8. Badimón L, Martínez-González J. Disfunción endotelial. Revista Española de Cardiología. 2006;6(Supl.A):2130. Available from: http://www.revespcardiol.org/es/ disfuncion-endotelial/articulo/13087918/.

9. Garay I, Olate S. Consideraciones Actuales en el Estudio Imagenológico de las Calcificaciones de Tejidos Blandos en Zona de Ángulo Mandibular. International journal of odontostomatology. 2013 Dec;7(3):455-464.

10. Guimarães Henriques JC. Panoramic Radiography in the Diagnosis of Carotid Artery Atheromas and the Associated Risk Factors. The Open Dentistry Journal. 2011 May;5(1):79-83. Available from: http://benthamopen. com/ABSTRACT/TODENTJ-5-79.

11. Monteiro IA, Ibrahim C, Albuquerque R, Donaldson N, Salazar F, Monteiro L. Assessment of carotid calcifications on digital panoramic radiographs: Retrospective analysis and review of the literature. Journal of Stomatology, Oral and Maxillofacial Surgery. 2018
Apr;119(2):102-106. Available from: http://linkinghub. elsevier.com/retrieve/pii/S2468785517302288.

12. Surur A, Cámara JP, Salvatierra W, Sanz R, Canavosio $\mathrm{N}$, Videla $\mathrm{R}$, et al. Localización $\mathrm{y}$ frecuencia de placas ateromatosas intracraneales en pacientes mayores de 40 años. Revista argentina de radiología. 2014 Dec;78(4):193-198. Available from: http://www. scielo.org.ar/scielo.php?script=sci_arttext\&pid=S185299922014000400002\&lng=en\&nrm=iso\&tlng=es.

13. Ibrahim Nasseh, Georges Aoun. Carotid Artery Calcification: A Digital Panoramic-Based Study. Diseases. 2018 Feb;6(1):15. Available from: http://www.mdpi.com/20799721/6/1/15.

14. Sposato LA, Riccio PM, Klein FR. Diagnóstico y tratamiento de la enfermedad carotídea aterosclerótica extracraneal asintomática. Medicina (Buenos Aires). 2011 Dec;71(6):561-565. Available from: http://www.scielo.org.ar/scielo.php?script=sci_arttext \& pid=S0025-76802011000800014.

15. Santos JMO, Soares GC, Alves APNN, Kurita LM, Silva PGdB, Costa FWG. Prevalence of carotid artery calcifications among 2,500 digital. Med Oral Patol Oral Cir Bucal. 2018 May;23(3):e256.

16. Brito ACRd, Nascimento HAR, Argento R, Beline T, Ambrosano GMB, Freitas DQ. Prevalence of suggestive images of carotid artery calcifications on panoramic radiographs and its relationship with predisposing factors. Ciência \& Saúde Coletiva. 2016 Jul;21(7):2201-2208. Available from: http://www.scielo.br/scielo.php?script=sci_arttext\& pid=S1413-81232016000702201\&lng=en\&tlng=en.

17. Moshfeghi M, Taheri JB, Bahemmat N, Evazzadeh ME, Hadian H. Relationship Between Carotid Artery Calcification Detected in Dental Panoramic Images and Hypertension and Myocardial Infarction. Iran J Radiol. 2014 Aug;11(3):e8714.

Recibido: 5 de febrero de 2018

Aceptado: 28 de mayo de 2018 
